\title{
Pratiques d'hygiène alimentaire dans les cantines en milieu hospitalier de Bamako
}

\section{Food hygiene practices in hospital canteens in Bamako}

\author{
TOURE $\mathrm{H}^{1}$, AG IKNANE $\mathrm{A}^{2}$, TOURE $0^{3}$.
}

1. Médecin de santé publique, spécialisée en santé communautaire, ANSSA, Bamako

2. Maître de conférnces en santé publique, DG ANSSA

3. Chercheur au département santé communautaire, INRSP, Bamako

\begin{abstract}
Résumé
Au Mali, Les hôpitaux sont des lieux privilégiés de ventes d'aliments de toutes sortes. C'est pour évaluer les pratiques d'hygiène alimentaire en milieu hospitalier que la présente étude a été réalisée.

Une étude transversale par sondage raisonné à l'aide d'un échantillonnage par commodité a été réalisée auprès de 30 restaurateurs et 59 Consommateurs du 3 au 5 Décembre 2012.

Les données avait été collecté auprès des restaurateurs publics et privés, des Consommateurs et des informateurs clés à l'aide d'un entretien directe semi- directif et d'une observation des pratiques au moyen d'une grille d'observation. Le test de chi ${ }^{2}$ de Pearson a été utilisé pour la comparaison des moyennes.
\end{abstract}

Nos résultats ont montré que seuls $16,7 \%$ des restaurateurs observaient les règles d'hygiène alimentaire. Près de 63,3\% d'entre eux ne désinfectaient pas les aliments préparés et les crudités ni à l'eau de javel ni au permanganate. Le lavage des mains au savon, était jugée acceptable chez $10 \%$ des enquêtés. Plus des deux tiers $(70 \%)$ de restaurateurs n'avaient reçu aucune formation sur les bonnes pratiques d'hygiène alimentaire. Pour $40 \%$ de consommateurs, l'environnement de préparation des aliments était sale et $66,7 \%$ des restaurateurs n'utilisaient pas de tenue de travail. Près de $28,8 \%$ des consommateurs avaient eu au moins une fois des malaises tels que diarrhée, douleurs abdominales et vomissements. L'hygiène alimentaire reste fortement influencée par le mode de préparation et le comportement des vendeurs qui devraient adopter de bonnes pratiques pour préserver la santé des consommateurs

Mots clés : Hygiène, aliments, restaurateurs,

\section{Summary}

In Mali, hospitals are the appropriate for the sales of food of any kind. .It is in order to evaluate food hygiene practice at hospitals that this present survey has been carried ant. A cross-sectional survey has been undertaken trough a descriptive pool by a convenient test in in30 (thirty) restaurants and 59 (fifty nine) Consumers, from 3 to 5 December

2012.

The relevant information has been collected from public and private restaurants, from, Consumers and key informers by a direct or semi structured interview and an observation through a chart. .The Pearson $\mathrm{Chi}^{2}$ test has been used to compare the averages.

Our results showed that only $16.7 \%$ of the restaurants were observing the food hygiene rules. Nearly $63.3 \%$ of them were not disinfecting prepared foods and raw vegetables with neither permanganate nor bleach. Washing hand in the soap has been judged acceptable by $10 \%$ of the interviewed.More than two over three $(70 \%)$ of the restaurant owners had not received any training about the good practices of food hygiene. For $40 \%$ of the consumers, the environment where food is prepared was dirty , and $66.7 \%$ of the restaurants were not using working clothes .Nearly $28.8 \%$ of the consumers had been at least once in trouble with illnesses such as diarrhea ,abdominal pain and vomiting. Food hygiene is remains strongly influenced by the way of cooking and the behavior of the, sellers who should adopt good practices to preserve the health of consumers

Key words: Health, Food, restaurant

\section{Introduction}

L'alimentation dans les cantines des hôpitaux est un phénomène quasi universel, rencontré en particulier en zone urbaine aussi bien dans les pays en développement qu'industrialisés. . Ce mode d'alimentation nécessite des conditions d'hygiène et d'assainissement devant permettre une protection optimale des consommateurs pour éviter les risques de toxi-infection. Tout contact avec un élément potentiellement contaminant, au sortir des toilettes, manger ou de préparer les repas, se laver les mains devient nécessaire pour éviter toute toxi-infection alimentaire. Le manque d'hygiène est incontestablement le principal responsable de l'incrimination de nos aliments, de nos milieux de vie, de nos propres corps comme réservoir de toutes sortes de maladies [1].

L'ignorance des vendeurs des aliments de rue est aussi un facteur qui aggrave les risques. Le manque d'hygiène, le manque d'eau potable, l'insalubrité des lieux de vente, la promiscuité avec les dépôts d'ordures et, les égouts d'évacuation des déchets, sont autant risques et de facteurs de préoccupations en terme de protection de la santé des populations. C'est dans l'optique d'évaluer le niveau de pratiques d'hygiène alimentaire dans les cantines des hôpitaux que la présente étude est réalisé à l'Hôpital Gabriel Touré.

\section{Matériel et méthodes}

II s'agissait d'une étude transversale, descriptive réalisée de novembre 2012 à février 2013. La population d'étude était composée d'une cible primaire, 30 restaurateurs des cantines (publique et privée) du CHU Gabriel TOURE et alentours et secondaire, composée de 59 consommateursdu CHU Gabriel TOURE et alentours, l'agent d'hygiène, du responsable du service social et du gérant de l'alimentation générale du $\mathrm{CHU}$ Gabriel TOURE.

La collecte des données a été réalisée à travers des entretiens individuels pour les différents informateurs clés. L'observation directe non participante à l'aide d'une grille a été réalisée au niveau des différentes cantines concernées et alentours. 
L'analyse des données a été réalisée à l'aide du logiciel SPSS 17.0 et le test de chi ${ }^{2}$ de Pearson a été utilisé pour la comparaison des moyennes avec un niveau de signification de $95 \%$.

\section{Résultats}

Le niveau de scolarisation des restaurateurs était de $46,6 \%$. Parmi les enquêtés, $30 \%$ n'avaient pas accédé au niveau primaire et $23,4 \%$ avaient atteint le secondaire. La grande majorité des restaurateurs étaient des femmes $(60 \%)$. Le niveau de connaissance des règles d'hygiène par les restaurateurs était de 40,0\% (Tableau I). Près de $83,3 \%$ de restaurateurs visités n'observaient pas les bonnes pratiques d'hygiène alimentaire (Tableau II). Près de $70 \%$ des restaurateurs n'avaient reçu aucune formation sur l'hygiène alimentaire. Les crudités n'avaient pas été désinfectées ni à l'eau de javel ni au permanganate dans $63,3 \%$ des cas.

L'environnement de préparation des aliments par les restaurateurs avait été jugé sale dans $40 \%$ des cas. Près de $46,7 \%$ de restaurateurs étaient installés dans des endroits insalubres aux abords des caniveaux, des eaux usées, des tas d'ordures. Les murs des restaurants étaient tapissés d'araignées dans $50 \%$ des cas et les tables à manger poussiéreuse dans $60 \%$ ) des cas. . Les aliments sont exposés à la poussière dans $63,3 \%$ et aux mouches dans $80,0 \%$. La conservation des aliments sur les lieux de distribution était mauvaise chez $30 \%$ des restaurateurs. Le lavage des mains au savon n'était pas pratiqué par $42,4 \%$ des restaurateurs Tableau III. Dans près de $20 \%$ des cas, les restes d'aliments étaient réchauffés et vendus à la clientèle. La collecte et la conservation des eaux usées se faisaient dans 33,3\% dans des récipients jusqu'à la fin de la journée. Près de $46,6 \%$ ) des restaurateurs (déversaient les eaux usées soit dans la rue, soit dans les caniveaux. La gestion des déchets ménagers était mauvaise chez $36,7 \%$ tableau IV des restaurateurs. Le lavage des mains avant la manipulation des aliments pendant la préparation des aliments, était insuffisant dans $56,7 \%$ et très insuffisant dans $33,3 \%$ des cas. L'occupation principale des restaurateurs avait une influence significative sur l'observance de la pratique de l'hygiène alimentaire $(p=0,041)$. Près de $28,8 \%$ des consommateurs avaient déclaré avoir eu au moins une fois, des malaises tels que la diarrhée, des douleurs abdominales et des vomissements, liés à la consommation des aliments provenant des restaurateurs du CHU Gabriel Touré. Plus de la moitié, soit $52,9 \%$ des consommateurs n'avaient pas une bonne appréciation des aliments des restaurateurs du CHU Gabriel Touré et alentours.

\section{Discussion}

Compte tenu de la taille de l'échantillon la puissance des tests statistiques a été diminuée.

Notre étude a montré que la plupart des restaurateurs du CHU Gabriel Touré et alentours étaient des femmes. Une étude réalisée au Mali en 2002 par l'ASCOMA avait déjà montré cette tendance [2].Une grande partie des restaurateurs $(46,6 \%)$ n'étaient pas scolarisé.. Ce faible niveau d'instruction pourrait expliquer la méconnaissance des règles élémentaires d'hygiène. Le faible niveau d'instruction est observé dans une étude menée en Afrique par la FAO en 1996 qui montre qu'en plus du faible niveau d'éducation, le manque d'emploi pousse la plupart des chômeurs à investir dans le secteur de l'alimentation de rue [3]. Dans notre étude près de $70 \%$ des enquêtés n'avaient reçu aucune formation sur l'hygiène alimentaire. De façon générale en Afrique de l'Ouest, les vendeuses d'aliments ne subissent aucune formation sur les bonnes pratiques de l'hygiène alimentaire. Leur installation est, dans la majorité des cas, informelle [4]. L'observation avait montré que les restaurateurs s'installaient parfois dans des endroits insalubres aux abords des caniveaux, des eaux usées. Beaucoup de restaurateurs exerçaient à côté des tas d'ordures $(46,7 \%)$, disposant de tables à manger poussiéreuse $(60 \%)$ avec des toiles d'araignées sur les murs dans $50 \%$ des cas. Les aliments sont exposés à la poussière dans $63,3 \%$ et aux mouches dans $80,0 \%$.

La pratique de l'hygiène chez ces restaurateurs reste mauvaise comme partout en Afrique [3]. Les restaurateurs n'ont aucune formation en hygiène alimentaire ou en assainissement et travaillent dans des conditions très rudimentaires et peu hygiéniques, ce qui pourrait provoquer des empoisonnements alimentaires dus à la consommation d'aliments insalubres. En janvier 2002, dans son rapport final sur l'alimentation de la rue au Mali, l'ASCOMA trouve que celle-ci devient de plus en plus la principale source nutritionnelle d'une frange considérable de la population citadine [2].L'occupation principale des restaurateurs a une influence sur l'observance de la pratique de l'hygiène alimentaire. Plus des deux tiers des consommateurs mangent tous les jours dans ces restaurants du CHU Gabriel et alentours. Des malaises tels que diarrhée $(6,80 \%)$, douleurs abdominales $(13,60 \%)$ et vomissements $(8,50 \%)$, liés à la consommation des aliments des restaurateurs du $\mathrm{CHU}$ Gabriel Touré et alentours ont été déclarés au moins une fois chez $28,8 \%$ des consommateurs.

\section{Conclusion}

De nos jours la préparation, la conservation, et la distribution des aliments sont fortement influencées par Le comportement des restaurateurs d'aliments.

La consommation d'aliments étant un besoin indispensable et vital pour l'être humain, elle devrait être accompagnée de mesures de sécurité.

\section{Références}

1-MAIGA B. Pratiques d'hygiène hospitalière dans les structures sanitaires : Hôpital Gabriel Touré, Hôpital Régional de Sikasso, CNOS, Centre de Santé Référence de la Commune IV de Bamako, Thèse de Médecine, FMPOS, Bamako, 2003.

2- ASCOMA. Etudes des aspects socio-économiques de la qualité et de la sécurité des aliments de rue dans les régions de Ségou, Sikasso et du District de Bamako, SADAOC, Bamako, 2002 pp (8 - 43).

3- FAO. Alimentation de rue en Afrique, FAO, 1996.

4- ASSONGBON Ekoué $M$. et al. Hygiène alimentaire et problématique de l'alimentation de rue en Afrique de l'Ouest, Sadoc infO, $2002 ; 1: 1-17$.

5. AG BENDECH M.Alimentation de rue à Bamako au Mali: problématique et approches d'intervention, Mali Santé Publique, 2013, pp : (44 - 47) 
TABLEAU II: Répartition des consommateurs du CHU Gabriel Touré et alentours en fonction de l'observance des mesures d'hygiene en 2012

\begin{tabular}{lccc}
\hline $\begin{array}{l}\text { Observance } \\
\text { mesures hygiène }\end{array}$ & Gérant & Employé & Total \\
\hline Non Observance & 22 & 3 & 25 \\
& $(91,7 \%)$ & $(50,0 \%)$ & $(83,3 \%)$ \\
Observance & 2 & 3 & 5 \\
& $(8,3 \%)$ & $(50,0 \%)$ & $(16,7 \%)$ \\
Total & 24 & 6 & 30 \\
& $(100,0 \%)$ & $(100,0 \%)$ & $(100,0 \%)$
\end{tabular}

TABLEAU III : Répartition des restaurateurs du CHU Gabriel Touré et alentours en fonction des pratiques d'hygiene en 2012

\begin{tabular}{lcc}
\hline $\begin{array}{c}\text { Pratiques d'hygiène } \\
\mathbf{n}=\mathbf{3 0}\end{array}$ & Effectif & Pourcentage \\
\hline $\begin{array}{l}\text { Propreté de l'environnement de préparation } \\
\text { des aliments }\end{array}$ & \\
Bonne & 5 & 16,6 \\
Acceptable & 13 & 43,3 \\
Mauvaise & 12 & $\mathbf{4 0 , 0}$ \\
Observance des règles d'hygiène & \\
Oui & 5 & 16,67 \\
Non & 25 & 83,33 \\
Pratique du lavage des mains & \\
Oui & 34 & 57,6 \\
Non & 25 & 42,4 \\
\hline
\end{tabular}

TABLEAU IV : Répartition des restaurateurs du CHU Gabriel Touré et alentours en fonction des mesures d'hygiène des aliments en 2012

\begin{tabular}{|c|c|c|}
\hline $\begin{array}{l}\text { Mesures d'hygiène } \\
n=30\end{array}$ & Effectif & Pourcentage \\
\hline \multicolumn{3}{|c|}{ Conservation des aliments sur les lieux } \\
\hline Bonne & 7 & 23,3 \\
\hline Acceptable & 14 & 46,7 \\
\hline Mauvaise & 9 & 30,0 \\
\hline \multicolumn{3}{|l|}{$\begin{array}{l}\text { Matériel de services des } \\
\text { aliments à emporter } \\
\text { (conditionnement) }\end{array}$} \\
\hline Réchauffés et vendus & 6 & 20 \\
\hline $\begin{array}{l}\text { Mangés en famille } \\
\text { Autres (gardiens, } \\
\text { chauffeurs del'hôpital, } \\
\text { les mendiants, les }\end{array}$ & 5 & 16,7 \\
\hline malades mentaux) & 19 & 63,3 \\
\hline \multicolumn{3}{|l|}{$\begin{array}{l}\text { Collecte et évacuation des } \\
\text { eaux usées }\end{array}$} \\
\hline Jetés dans la rue & 7 & 23,3 \\
\hline $\begin{array}{l}\text { Conservées dans des } \\
\text { récipients }\end{array}$ & 10 & 33,3 \\
\hline Caniveaux & 7 & 20,0 \\
\hline $\begin{array}{l}\text { Autres (lieu de } \\
\text { préparation des aliments) }\end{array}$ & 6 & 23,3 \\
\hline \multicolumn{3}{|c|}{ Gestion des déchets ménagers } \\
\hline Bonne & 3 & 10,0 \\
\hline Acceptable & 16 & 53,3 \\
\hline Mauvaise & 11 & 36,7 \\
\hline
\end{tabular}

